\title{
Dietary flavonoid intake and colorectal cancer: a case-control study
}

\author{
Janet A. M. Kyle ${ }^{1,2}$, Linda Sharp ${ }^{3,4}$, Julian Little ${ }^{3,5}$, Garry G. Duthie ${ }^{1}$ and Geraldine McNeill ${ }^{2}$ \\ ${ }^{1}$ Rowett Institute of Nutrition and Health, University of Aberdeen, Aberdeen AB21 9SB, UK \\ ${ }^{2}$ Institute of Applied Health Sciences, University of Aberdeen, Aberdeen AB25 2ZD, UK \\ ${ }^{3}$ Epidemiology Group, University of Aberdeen, Aberdeen AB25 2ZD, UK \\ ${ }^{4}$ National Cancer Registry Ireland, Elm Court, Boreenmanna Road, Cork, Republic of Ireland \\ ${ }^{5}$ Canada Research Chair in Human Genome Epidemiology, Department of Epidemiology and Community Medicine, \\ University of Ottawa, Ottawa, Canada
}

(Received 25 February 2009 - Revised 21 July 2009 - Accepted 27 July 2009 - First published online 7 September 2009)

Diets rich in flavonoids may reduce the risk of developing colorectal cancer. Flavonoids are widely distributed in foods of plant origin, though in the UK tea is the main dietary source. Our objective was to evaluate any independent associations of total dietary and non-tea intake of four flavonoid subclasses and the risk of developing colorectal cancer in a tea-drinking population with a high colorectal cancer incidence. A population-based case-control study (264 cases with histologically confirmed incident colorectal cancer and 408 controls) was carried out. Dietary data gathered by FFQ were used to calculate flavonoid intake. Adjusted OR and $95 \%$ CI were estimated by logistic regression. No linear association between risk of developing colorectal cancer and total dietary flavonol, procyanidin, flavon-3-ol or flavanone intakes was found, but non-tea flavonol intake was inversely associated with colorectal cancer risk (OR 0.6; $95 \%$ CI 0.4, 1.0). Stratification by site of cancer and assessment of individual flavonols showed a reduced risk of developing colon but not rectal cancer with increasing non-tea quercetin intake (OR $0.5 ; 95 \%$ CI 0.3, 0.8; $\left.P_{\text {trend }}<0.01\right)$. We concluded that flavonols, specifically quercetin, obtained from non-tea components of the diet may be linked with reduced risk of developing colon cancer.

Flavonoids: Flavonols: Quercetin: Colorectal cancer: Case-control studies: Epidemiology

Colorectal cancer is the third most common cancer in the developed world ${ }^{(1)}$ with incidence in Scotland being among the highest in Europe ${ }^{(2)}$. Critical assessment of potential risk factors suggests that diets rich in plant-based foods, such as fruit and vegetables, may reduce the risk of developing colorectal cancer ${ }^{(3,4)}$. Though the mechanism by which these foods exert a protective effect is unclear, one hypothesis is the presence of high levels of potentially anti-carcinogenic phytochemicals ${ }^{(5)}$. Flavonoids are a large and diverse group of phytochemicals and research into their anti-carcinogenic potential with animal and cellular model systems supports a protective role ${ }^{(6,7)}$. Structurally distinct subclasses of flavonoids have varying capacities to modulate the progression of colorectal cancer, acting as antioxidants ${ }^{(8,9)}$, anti-inflammatory agents ${ }^{(10-13)}$, anti-proliferative agents ${ }^{(14-16)}$ or as regulators of signal transduction pathways ${ }^{(17,18)}$. Of all the tissues in the human body, the large intestine may be exposed to higher flavonoid concentrations than other tissues ${ }^{(19)}$.

Epidemiological assessment of the relationship between dietary flavonoid intake and colorectal cancer is limited, with different cohort studies investigating different combinations of flavonoids ${ }^{(20-25)}$. With the exception of the Iowa Women's Health study ${ }^{(25)}$, where a significant inverse association between catechin intake and incidence of rectal cancer was found $(P<0 \cdot 01)$, no significant relationships between flavonoid intake and colorectal cancer incidence have been reported. Recently, two case-control studies utilised new and relatively comprehensive flavonoid food composition databases $^{(26,27)}$ examining potential beneficial links between flavonoid intake and development of colorectal cancer. An Italian case-control study ${ }^{(28)}$ found reduced risk of developing colorectal cancer with increasing isoflavone, anthocyanin, flavone and flavonol intakes, but not with catechin or flavanone intake. A large Scottish case-control study $^{(29)}$ also observed reduced colorectal cancer risk with higher intakes of flavonols, catechins and procyanidins type B1-B4, though not with flavanones or phyto-oestrogens.

Black tea is an important source of flavonoids. It is likely that the distribution of flavonoid subclasses differs in different populations depending on the relative intakes of fruit, vegetables and tea. The typical Scottish diet is known to be low in fruit and vegetables and high in processed foods, with black tea commonly consumed ${ }^{(30,31)}$. Dietary flavonoid intakes in a North East of Scotland population were found to be comparable with other European countries including Italy ${ }^{(26)}$. Unlike the Scottish diet, high fruit and vegetable intake and low tea consumption characterise the Italian $\operatorname{diet}^{(32)}$. Consequently, the observation of comparable levels 
of flavonoid intake may be misleading, with black tea being the major source of flavonoids in the Scottish population ${ }^{(26)}$. Additionally, when assessing associations between flavonoid intake and disease risk in populations where high levels of tea are commonly consumed, misclassification of risk may occur if non-tea dietary sources are neglected ${ }^{(24)}$.

The present study investigated the associations between dietary flavonoid intake and the risk of developing colorectal cancer in the North East of Scotland. The aim was to assess whether this relationship varied when considering total dietary and non-tea intake of four flavonoid subclasses (flavonols, catechins (flavon-3-ols), procyanidins and flavanones).

\section{Materials and methods}

\section{Subjects and study design}

Participants were recruited as part of a population-based case-control study of colorectal cancer investigating colorectal cancer and genetic polymorphisms in xenobiotic metabolising enzymes in the North East of Scotland ${ }^{(33)}$. Patients (cases) presenting with their first primary cancer, diagnosed between September 1998 and February 2000, and resident in the Grampian health board area were approached after histological confirmation of incident invasive tumours of the colon or rectum. Population-based controls, frequency matched to cases, were selected from the Grampian Community Health Index (a list of all those registered with a general practitioner in the National Health Service). Controls who declined to participate were replaced. The present study was conducted according to the guidelines laid down in the declaration of Helsinki and all procedures involving human subjects and patients were approved by the Joint Ethical Committee of the Grampian Health Board and the University of Aberdeen. Written consent was obtained from all participants.

\section{Dietary assessment and analysis}

With the permission of their general practitioner, subjects were contacted by mail and asked to complete the Scottish Collaborative Group FFQ version 6.31 (www.foodfrequency.org.uk) and a questionnaire which included questions on a range of sociodemographic and lifestyle factors relevant to colorectal cancer aetiology. Of those invited to participate, $62 \%$ of cases and $61 \%$ of controls completed and returned our questionnaires (264 cases and 408 controls). Of these, seven subjects (three cases and four controls) were excluded as their FFQ were returned incomplete ${ }^{(33)}$.

Dietary data were converted into estimates of nutrient intakes using the UK McCance and Widdowson's food composition tables ${ }^{(34)}$. Twelve subjects (six cases and six controls) were excluded on the basis of implausible total energy intakes ( \pm 3 SD of mean intake $)^{(35)}$. Total dietary and non-tea flavonol, flavon-3-ol, procyanidin and flavanone intakes were computed using a flavonoid food composition database compiled by systematic review of the available composition literature representing foods consumed in the $\mathrm{UK}^{(26)}$. In this database, as numerous conjugated forms of flavonoids are commonly present in foods, all food content and intake values are expressed in their free (aglycone) form. Additionally, each subclass is a summary of individual flavonoid compounds as outlined: flavonols - quercetin, kaempferol and myricetin; flavones - lutein and apigenin; flavon-3-ols - catechin, epicatechin, epigallocatechin, epigallocatechin gallate, epicatechin gallate and gallocatechin; procyanidins - types BI-IV; flavanones - hesperidin and naringenin. The 150-item semi-quantitative FFQ was previously tested in the local population and compared with $4 \mathrm{~d}$ weighed intake records for macro- and micronutrients as well as each flavonoid subclass ${ }^{(35,36)}$. Relatively good Spearman rank correlation and agreements were found for flavonols, procyanidins and flavon-3-ols (correlations 0.70-0.94), but not for flavanones and flavones, $0 \cdot 33$ and $0 \cdot 18$ respectively. As a result, flavone data were not used. The FFQ included questions on frequency of consumption of each food item in the last year as 'rarely or never', 'monthly' or 1, 2, 3, 4, 5, 6, $7 \mathrm{~d}$ per week and amount ('measures') of consumption of a range of food items. The measure for each food was designed to be a small portion or unit measure such as a tablespoon; hence a single standard portion of a particular food might be two measures ${ }^{(36)}$.

\section{Statistical analysis}

SPSS (version 13; SPSS, Inc., Chicago, IL, USA) and Stata 8 (StataCorp LP, College Station, TX, USA) were used for statistical analysis. Case and control demographic and lifestyle factors were summarised. Independent $t$ tests and $\chi^{2}$ tests assessed the significance of any differences. The mean values and standard deviations of measures of tea, fruit and vegetables were calculated. The measure for tea was a cup, while fruit and vegetable measures were the total number of single portions per $\mathrm{d}$ as reported on the FFQ.

The distributions of flavonoid variables were checked. Log transformation normalised the non-tea flavonoid intake, but not total flavanone intake. Median intake and interquartile range of flavonoid subclasses and individual compounds were computed for cases and controls before investigating any significant differences by applying the non-parametric Wilcoxon rank-sum test. The correlation between flavonoid intakes and other dietary and non-dietary factors (for example, $\mathrm{BMI}$ ) were calculated using the Spearman rank correlation coefficients.

Flavonoid, tea, fruit and vegetable intakes were adjusted for total energy using the nutrient residual method ${ }^{(37)}$ before division of subjects into quartiles of intake (lowest to highest). The odds of developing colorectal cancers with increasing intake of flavonoids were then computed. Multivariate adjusted OR were adjusted for sex, age, correlated dietary variables and potential confounders; factors making a significant contribution (likelihood ratio test $P<0 \cdot 1$ ) were retained in the regression model, including variables with significant correlations with flavonoid intake. The likelihood ratio test was used to assess trends across the quartiles of flavonoid intake $\left(P_{\text {trend }}\right)$. The primary analysis was for all colorectal cancers combined; secondary analyses were carried out for colon and rectal cancers separately.

\section{Results}

A total of 261 colorectal cancer patients (cases) (186 and seventy-five with colon and rectal cancer, respectively) 
and 404 population-based control subjects were included in the full analysis of the present investigation (Table 1). Cases (age range 39-92 years) tended to be older than controls (age range 32-88 years) and a higher proportion of cases were male. Although fewer cases were current smokers $(P<0.01)$, more were ex-smokers $(P<0.01)$ than controls. A higher percentage of cases reported a family history of colorectal cancer compared with controls, while non-steroidal anti-inflammatory drugs were more frequently taken by controls.

When stratified by sex, energy intake of cases and controls did not differ significantly, though energy intake in men $(10 \cdot 5$ (SD 3.8) $\mathrm{MJ} / \mathrm{d}$ ) was significantly higher than in women (9.1 (SD 3.9) $\mathrm{MJ} / \mathrm{d}$ ) for both cases and controls $(P<0.05)$. Significantly higher total dietary intake of flavonols, procyanidins and flavanones was recorded for cases than controls. The converse was observed for non-tea flavonol, procyanidin and flavon-3-ol intake, with controls reporting higher intakes (Table 2).

Tea intake was highly correlated with flavonol, procyandin and flavon-3-ol intakes $(r>0.90 ; P<0 \cdot 001)$. Exclusion of tea as a potential source of flavonoids yielded markedly lower estimates of intake (Table 2), with black tea consumption accounting for between 71 and $93 \%$ of total flavonol, procyanidin and flavon-3-ol intakes. After exclusion of tea flavonoids, gallated catechin esters (epigallocatechin gallate, epicatechin gallate and epigallocatechin) were replaced with catechin and epicatechin as the main contributors to dietary flavon-3-ol intake, while quercetin remained the main flavonol consumed before and after adjustment for tea consumption.

Colorectal cancer patients consumed more tea than control subjects $(P<0.01)$, drinking an average of 3.7 (SD 0.1$)$ and $3 \cdot 3$ (SD 0.1) cups per d, respectively. There were no significant differences between reported fruit and vegetable intake between cases and controls (1.9 (SD 1.8) and 1.9 (SD 1.9) measures of fruit per $d$ and 3.1 (SD 4.2) and 3.6 (SD 3.9) measures of vegetables per $\mathrm{d}$ for cases and controls,

Table 1. Demographic characteristics of cases and controls

\begin{tabular}{|c|c|c|}
\hline & Cases $(n 261)$ & Controls ( $n$ 404) \\
\hline \multicolumn{3}{|l|}{ Age (years) } \\
\hline Mean & $69 \cdot 8^{\star \star \star}$ & $63 \cdot 0$ \\
\hline SD & $10 \cdot 5$ & $11 \cdot 2$ \\
\hline Sex (\% male) & 57 & 52 \\
\hline \multicolumn{3}{|l|}{ BMI $\left(\mathrm{kg} / \mathrm{m}^{2}\right)$} \\
\hline Mean & $26 \cdot 3$ & $25 \cdot 9$ \\
\hline SD & $10 \cdot 7$ & 4.4 \\
\hline \multicolumn{3}{|l|}{ Smoking (\%) } \\
\hline Never & 41 & 42 \\
\hline Ex-smoker & $46^{\star}$ & 39 \\
\hline Current & $9^{*}$ & 16 \\
\hline $\begin{array}{l}\text { Family history of colorectal } \\
\text { cancer (\% yes) }\end{array}$ & $20^{\star *}$ & 8 \\
\hline \multicolumn{3}{|l|}{ Site of cancer } \\
\hline Colon $(n)$ & 186 & - \\
\hline Rectum ( $n$ ) & 75 & - \\
\hline Aspirin (\% taking) & 20 & 22 \\
\hline NSAID (\% taking) & $13^{\star}$ & 22 \\
\hline
\end{tabular}

NSAID, non-steroidal anti-inflammatory drugs.

Value was significantly different from that of the controls: ${ }^{\star} P<0.05,{ }^{\star \star} P<0.01$, ${ }^{* * *} P<0.001$. respectively). Controls under the age of 55 years consumed less tea and more vegetables than controls over 55 years $(P<0 \cdot 01)$, but this effect was not seen in cases. This had an impact on intake of both total and non-tea flavonoids, with controls reporting a lower intake of flavonols $(P<0.05)$ and a higher intake of procyanidins $(P<0.05)$ and flavon-3-ols $(P<0 \cdot 01)$ than cases.

Increasing energy-adjusted black tea consumption demonstrated a weak increased risk of developing colorectal cancer (OR 1.5; $95 \%$ CI 1.0, 2.4; highest $v$. lowest quartile; $\left.P_{\text {trend }}=0.08\right)$, but this was attenuated after adjustment for confounding variables. Energy-adjusted vegetable intake was significantly associated with colon cancer (highest quartile of energy-adjusted intake OR $0.6 ; 95 \%$ CI $0.3,0.9$; $\left.P_{\text {trend }}=0.03\right)$. Again this association was not seen after multivariate adjustment (highest $v$. lowest quartile of energy-adjusted intake OR $1.0 ; 95 \%$ CI $0.6, \quad 1.8$; $P_{\text {trend }}=0 \cdot 52$ ). No effect was seen with rectal cancer. There was also no evidence of a relationship between fruit consumption and colorectal cancers (data not shown).

No association between total dietary flavonol, procyanidin or flavon-3-ol intake and risk of developing colorectal cancer was observed (Table 3). There was a weak $\left(P_{\text {trend }}=0.04\right)$ trend towards increased risk of colorectal cancer with higher levels of flavanone intake. Stratification by cancer site (Table 4) strengthened this observation, with a significant trend apparent for colon cancer (multivariate OR $1.3 ; 95 \%$ CI $0.7,2.4$; highest $v$. lowest quartile; $\left.P_{\text {trend }}<0 \cdot 01\right)$.

Analysis of non-tea flavonoid intake indicated a significant inverse association between non-tea flavonol intake and risk of colorectal cancer $(P<0 \cdot 05)$, but not for non-tea procyanidin or flavon-3-ol intake (Table 3). Separate analyses of colon and rectal cancer cases demonstrated that non-tea flavonol intake was significantly associated with a reduced risk of developing colon (OR 0.5; $95 \%$ CI 0.3, 0.8; highest $v$. lowest quartile; $\left.P_{\text {trend }}<0 \cdot 01\right)$, but not rectal cancer in the adjusted model (Table 4). Further assessment of the relationship with intake of individual non-tea flavonol compounds highlighted an association between quercetin (highest $v$. lowest quartile multivariate adjusted OR $0.4 ; 95 \%$ CI $0.2, \quad 0 \cdot 8$; $\left.P_{\text {trend }}<0 \cdot 01\right)$ and colon cancer (Table 5).

\section{Discussion}

Associations between dietary intake of four different flavonoid subclasses and colorectal cancer risk were assessed in the present case-control study amongst men and women from the North East of Scotland. Total dietary flavonol, flavan-3-ol or procyanidin intakes were not associated with colorectal cancer risk. However, there was a weak negative trend with flavanone intake and colon cancer risk. Black tea and its flavonoids did not appear to be related to colorectal cancer risk in this population. An inverse association between colorectal cancers, more specifically colon cancer, and non-tea quercetin intake was observed.

While the retrospective nature of the case-control design of the present study makes it potentially susceptible to recall bias, the fact that the study has a population-based study design helps to minimise selection biases. A similar participation rate was achieved as in a previous UK postal 
Table 2. Dietary flavonoid intakes of cases and controls (Medians and interquartile ranges (IQR))

\begin{tabular}{|c|c|c|c|c|c|c|c|c|}
\hline \multirow[b]{3}{*}{ Flavonoid } & \multicolumn{4}{|c|}{ Total dietary intake $(\mathrm{mg} / \mathrm{d})$} & \multicolumn{4}{|c|}{ Non-tea dietary intake $(\mathrm{mg} / \mathrm{d})$} \\
\hline & \multicolumn{2}{|c|}{ Cases } & \multicolumn{2}{|c|}{ Controls } & \multicolumn{2}{|c|}{ Cases } & \multicolumn{2}{|c|}{ Controls } \\
\hline & Median & IQR & Median & IQR & Median & IQR & Median & IQR \\
\hline Flavonols & $32 \cdot 5^{\star \star *}$ & $22 \cdot 6-40 \cdot 3$ & $28 \cdot 6$ & $17 \cdot 4-40 \cdot 5$ & $7 \cdot 6^{\star * *}$ & $5 \cdot 3-10 \cdot 9$ & $8 \cdot 3$ & $5 \cdot 7-11 \cdot 0$ \\
\hline Quercetin & $20 \cdot 2$ & $14 \cdot 7-24.9$ & $18 \cdot 1$ & $11 \cdot 9-25 \cdot 0$ & $6 \cdot 6^{* * *}$ & $4 \cdot 6-9.5$ & $7 \cdot 1$ & $4 \cdot 9-9 \cdot 6$ \\
\hline Kaempferol & $10 \cdot 2^{*}$ & $5 \cdot 7-12 \cdot 9$ & $8 \cdot 0$ & $5 \cdot 0-12 \cdot 8$ & 0.8 & $0.5-1.1$ & $0 \cdot 8$ & $0.5-1.1$ \\
\hline Myricetin & $2 \cdot 1^{\star \star}$ & $5 \cdot 7-12 \cdot 9$ & 1.9 & $1 \cdot 1-2 \cdot 8$ & 0.2 & $0.0-0.4$ & 0.2 & $0.1-0.4$ \\
\hline Procyanidins B1-B4 & $39 \cdot 3^{\star \star \star}$ & $25 \cdot 8-49 \cdot 3$ & $34 \cdot 1$ & $19 \cdot 2-50 \cdot 0$ & $4 \cdot 8^{\star \star \star}$ & $2 \cdot 3-8 \cdot 5$ & $5 \cdot 2$ & $2 \cdot 5-9 \cdot 6$ \\
\hline Catechins & $141 \cdot 0^{\star \star}$ & $83 \cdot 6-180 \cdot 6$ & $119 \cdot 2$ & $73 \cdot 3-188 \cdot 8$ & $8 \cdot 2^{\star}$ & $4 \cdot 0-13 \cdot 3$ & 8.5 & $4 \cdot 0-13 \cdot 0$ \\
\hline (-)-Epigallocatechin & $25 \cdot 7$ & $12 \cdot 9-32 \cdot 2$ & $19 \cdot 3$ & $6 \cdot 7-32 \cdot 2$ & 0.2 & $0.0-0.4$ & $0 \cdot 1$ & $0.0-0.0$ \\
\hline$(+)$-Catechin & $7 \cdot 5^{\star \star}$ & $5 \cdot 5-9 \cdot 8$ & $6 \cdot 7$ & $4 \cdot 7-9 \cdot 6$ & $2 \cdot 4^{*}$ & $1 \cdot 2-4 \cdot 0$ & $2 \cdot 4$ & $1 \cdot 2-4.0$ \\
\hline (-)-Epicatechin & $24 \cdot 6$ & $20 \cdot 0-32 \cdot 1$ & $25 \cdot 3$ & $14 \cdot 2-36 \cdot 6$ & $5 \cdot 4^{*}$ & $2 \cdot 4-8 \cdot 2$ & $5 \cdot 6$ & $2 \cdot 5-8 \cdot 7$ \\
\hline (-)-Epigallocatechin gallate & $34 \cdot 6$ & $19 \cdot 8-42 \cdot 5$ & $29 \cdot 7$ & $9 \cdot 9-49 \cdot 5$ & 0.0 & $0.0-0.0$ & 0.0 & $0.0-0.0$ \\
\hline (-)-Epicatechin gallate & $39 \cdot 0$ & $19 \cdot 6-48 \cdot 8$ & $29 \cdot 2$ & $109-48 \cdot 7$ & 0.0 & $0.0-0.0$ & 0.0 & $0.0-0.0$ \\
\hline (-)-Gallocatechin & $12 \cdot 5^{\star}$ & $6 \cdot 3-15 \cdot 7$ & $9 \cdot 4$ & $3 \cdot 5-15 \cdot 7$ & $0 \cdot 1$ & $0.0-0.0$ & 0.1 & $0.0-0 \cdot 1$ \\
\hline Flavanones & $19 \cdot 0^{\star \star \star}$ & $5 \cdot 4-36 \cdot 4$ & $13 \cdot 4$ & $2 \cdot 7-32 \cdot 1$ & - & - & - & - \\
\hline Naringenin & $8 \cdot 9^{\star \star \star}$ & $2 \cdot 5-16 \cdot 7$ & $6 \cdot 6$ & $1 \cdot 4-15 \cdot 2$ & - & - & - & - \\
\hline Hesperidin & $9 \cdot 8^{\star * *}$ & $3 \cdot 0-19 \cdot 3$ & $7 \cdot 2$ & $1 \cdot 4-16 \cdot 6$ & - & - & - & - \\
\hline
\end{tabular}

Median was significantly different from that of the controls: ${ }^{*} P<0.05,{ }^{* *} P<0.01,{ }^{* *} P<0.001$.

contact study ${ }^{(38)}$. In addition, response rates and the quality of completion of the dietary questionnaires in the cases and controls were comparable. Moreover, it is worth noting that assessment of known risk factors resulted in associations consistent with previous evidence ${ }^{(33)}$, while reported energy intake was comparable with previous observations for this population. Multiple testing may also be a potential source of bias, though due to the relatively small sample size, the present study's aim was hypothesis generating rather than hypothesis testing.

Table 3. Total dietary and non-tea flavonoid intakes by quartile of intake and risk of colorectal cancer (261 cases and 404 controls) (Odds ratios and $95 \%$ confidence intervals)

\begin{tabular}{|c|c|c|c|c|c|c|c|c|c|c|}
\hline & \multicolumn{5}{|c|}{ Total dietary flavonoids } & \multicolumn{5}{|c|}{ Non-tea flavonoids } \\
\hline & \multirow[b]{2}{*}{ Intake (mg) } & \multicolumn{2}{|c|}{ Energy adjusted† } & \multicolumn{2}{|c|}{$\begin{array}{l}\text { Multivariate } \\
\text { adjusted } \neq\end{array}$} & \multirow[b]{2}{*}{ Intake (mg) } & \multicolumn{2}{|c|}{ Energy adjusted $†$} & \multicolumn{2}{|c|}{$\begin{array}{c}\text { Multivariate } \\
\text { adjusted } \neq\end{array}$} \\
\hline & & OR & $95 \% \mathrm{Cl}$ & OR & $95 \% \mathrm{Cl}$ & & OR & $95 \% \mathrm{Cl}$ & OR & $95 \% \mathrm{Cl}$ \\
\hline \multicolumn{11}{|c|}{ Flavonols } \\
\hline 1 & $<19.30$ & $1 \cdot 0$ & Reference & 1.0 & Reference & $<5.60$ & $1 \cdot 0$ & Reference & $1 \cdot 0$ & Reference \\
\hline 2 & $19 \cdot 31-30 \cdot 40$ & $1 \cdot 2$ & $0.7,1.9$ & $1 \cdot 0$ & $0.6,1.7$ & $5 \cdot 61-8 \cdot 00$ & 0.8 & $0.5,1.2$ & 0.8 & $0.5,1.3$ \\
\hline 3 & $30 \cdot 41-40 \cdot 40$ & 1.5 & $1 \cdot 0,2 \cdot 3$ & $1 \cdot 3$ & $0 \cdot 8,2 \cdot 1$ & $8 \cdot 01-10 \cdot 98$ & 0.7 & $0.4,1 \cdot 1$ & 0.7 & $0.4,1 \cdot 1$ \\
\hline 4 & $>40.41$ & 1.0 & $0 \cdot 6,1 \cdot 6$ & 0.8 & $0.5,1 \cdot 3$ & $>10.99$ & $0.6^{\star}$ & $0.4,1 \cdot 0^{\star}$ & 0.6 & $0.4,1.0$ \\
\hline$P_{\text {trend }}$ & & \multicolumn{2}{|c|}{0.20} & \multicolumn{2}{|c|}{0.37} & & \multicolumn{2}{|c|}{0.03} & \multicolumn{2}{|c|}{0.03} \\
\hline \multicolumn{11}{|c|}{ Procyanidins B1-B4 } \\
\hline 1 & $<21 \cdot 30$ & $1 \cdot 0$ & Reference & $1 \cdot 0$ & Reference & $<2.39$ & $1 \cdot 0$ & Reference & $1 \cdot 0$ & Reference \\
\hline 2 & $21 \cdot 31-36 \cdot 40$ & $1 \cdot 1$ & $0.7,1.7$ & 0.9 & $0.6,1.5$ & $2.40-5.06$ & 1.5 & $1 \cdot 0,2 \cdot 3$ & $1 \cdot 7^{*}$ & $1 \cdot 1,2 \cdot 8$ \\
\hline 3 & $36 \cdot 41-49 \cdot 80$ & 1.4 & $0.9,2 \cdot 1$ & $1 \cdot 2$ & $0.7,1.9$ & $5 \cdot 07-9 \cdot 25$ & 0.8 & $0.5,1 \cdot 3$ & $1 \cdot 0$ & $0 \cdot 6,1 \cdot 7$ \\
\hline 4 & $>49 \cdot 81$ & 0.9 & $0.6,1.4$ & 0.7 & $0.4,1.2$ & $>6.26$ & 0.9 & $0.6,1.4$ & $1 \cdot 2$ & $0 \cdot 7,2 \cdot 1$ \\
\hline$P_{\text {trend }}$ & & \multicolumn{2}{|c|}{0.1} & \multicolumn{2}{|c|}{0.19} & & \multirow{2}{*}{\multicolumn{2}{|c|}{0.17}} & \multirow{2}{*}{\multicolumn{2}{|c|}{0.17}} \\
\hline Flavono & & & & & & & & & & \\
\hline 1 & $<67 \cdot 10$ & $1 \cdot 0$ & Reference & $1 \cdot 0$ & Reference & $<4.00$ & $1 \cdot 0$ & Reference & $1 \cdot 0$ & Reference \\
\hline 2 & $67 \cdot 11-119 \cdot 20$ & 0.8 & $0.5,1.2$ & 0.7 & $0.4,1 \cdot 1$ & $4.01-8.29$ & 0.9 & $0.6,1.4$ & $1 \cdot 1$ & $0.7,1.7$ \\
\hline 3 & $119 \cdot 21-188 \cdot 80$ & $1 \cdot 7^{\star}$ & $1 \cdot 1,2 \cdot 7$ & $1 \cdot 3$ & $0.8,2 \cdot 2$ & $8 \cdot 30-12.99$ & $1 \cdot 1$ & $0.7,1.6$ & $1 \cdot 2$ & $0.8,2 \cdot 0$ \\
\hline 4 & $>188.81$ & 0.8 & $0.5,1 \cdot 3$ & 0.6 & $0.4,1.0$ & $>13.00$ & $1 \cdot 2$ & $0.8,2 \cdot 0$ & 1.0 & $0.6,1.8$ \\
\hline$P_{\text {trend }}$ & & \multicolumn{2}{|c|}{0.17} & \multicolumn{2}{|c|}{0.17} & & \multicolumn{2}{|c|}{0.08} & \multicolumn{2}{|c|}{0.25} \\
\hline \multicolumn{11}{|c|}{ Flavanones } \\
\hline 1 & $<2.73$ & 1.0 & Reference & 1.0 & Reference & & & & & \\
\hline 2 & $2 \cdot 74-13 \cdot 40$ & $1 \cdot 2$ & $0.8,2 \cdot 0$ & 1.5 & $0.9,2.5$ & - & & - & & - \\
\hline 3 & $13 \cdot 41-32 \cdot 18$ & $1 \cdot 2$ & $0.8,1.9$ & 1.4 & $0.9,2.4$ & - & & - & & - \\
\hline 4 & $>32.19$ & $1 \cdot 3$ & $0 \cdot 9,2 \cdot 1$ & 1.6 & $1 \cdot 0,2 \cdot 6$ & - & & - & & - \\
\hline$P_{\text {trend }}$ & & \multicolumn{2}{|c|}{0.07} & \multicolumn{2}{|c|}{0.04} & - & & - & & - \\
\hline
\end{tabular}

${ }^{*} P<0.05$.

$\dagger$ Energy adjusted.

$\ddagger$ Adjusted for energy, age at diagnosis, family history, non-steroidal anti-inflammatory drugs, aspirin, Mn, riboflavin, vitamin C, folate. 
Table 4. Association between total dietary and non-tea flavonoid intakes by quartile of intake and colon and rectal cancers $\dagger$ (Odds ratios and $95 \%$ confidence intervals)

\begin{tabular}{|c|c|c|c|c|c|c|c|c|}
\hline \multirow{3}{*}{ Flavonoids... } & \multicolumn{4}{|c|}{ Colon cancer (186 cases) } & \multicolumn{4}{|c|}{ Rectal cancer ( 75 cases) } \\
\hline & \multicolumn{2}{|c|}{ Total dietary } & \multicolumn{2}{|c|}{ Non-tea } & \multicolumn{2}{|c|}{ Total dietary } & \multicolumn{2}{|c|}{ Non-tea } \\
\hline & OR & $95 \% \mathrm{Cl}$ & OR & $95 \% \mathrm{Cl}$ & OR & $95 \% \mathrm{Cl}$ & OR & $95 \% \mathrm{Cl}$ \\
\hline \multicolumn{9}{|l|}{ Flavonols } \\
\hline 1 & 1.0 & Reference & 1.0 & Reference & 1.0 & Reference & $1 \cdot 0$ & Reference \\
\hline 2 & 0.9 & $0.5,1.6$ & 0.7 & $0 \cdot 4,1 \cdot 1$ & 1.2 & $0 \cdot 6,2 \cdot 6$ & $1 \cdot 1$ & $0 \cdot 5,2 \cdot 2$ \\
\hline 3 & 1.3 & $0.9,2 \cdot 1$ & 0.7 & $0.4,1.2$ & 1.1 & $0.5,2.2$ & 0.5 & $0.2,1 \cdot 1$ \\
\hline 4 & 0.7 & $0.4,2 \cdot 1$ & $0.5^{\star}$ & $0.3,0.8$ & $1 \cdot 1$ & $0.5,2.3$ & $1 \cdot 1$ & $0.5,2.4$ \\
\hline$P_{\text {trend }}$ & \multicolumn{2}{|c|}{0.54} & \multicolumn{2}{|c|}{0.01} & \multicolumn{2}{|c|}{0.96} & \multicolumn{2}{|c|}{0.52} \\
\hline \multicolumn{9}{|c|}{ Procyanidins B1-B4 } \\
\hline 1 & 1.0 & Reference & 1.0 & Reference & 1.0 & Reference & 1.0 & Reference \\
\hline 2 & $1 \cdot 1$ & $0.6,1.8$ & $2 \cdot 1^{*}$ & $1.2,3.5$ & 0.8 & $0.4,1 \cdot 1$ & $1 \cdot 2$ & $0.6,2.5$ \\
\hline 3 & 1.3 & $0.8,2 \cdot 2$ & $1 \cdot 2$ & $0.7,2.2$ & 0.8 & $0.4,1.6$ & 0.7 & $0.3,1.5$ \\
\hline 4 & 0.7 & $0.4,1.2$ & 1.4 & $0.7,2.5$ & 0.8 & $0.4,1.6$ & $1 \cdot 1$ & $0.5,2.3$ \\
\hline$P_{\text {trend }}$ & \multicolumn{2}{|c|}{0.24} & \multicolumn{2}{|c|}{0.99} & \multicolumn{2}{|c|}{0.79} & \multicolumn{2}{|c|}{0.71} \\
\hline \multicolumn{9}{|l|}{ Flavon-3-ols } \\
\hline 1 & 1.0 & Reference & 1.0 & Reference & 1.0 & Reference & 1.0 & Reference \\
\hline 2 & 0.8 & $0.4,1 \cdot 2$ & 1.3 & $0 \cdot 8,2 \cdot 1$ & 0.6 & $0.3,1.2$ & $1 \cdot 1$ & $0.5,2 \cdot 2$ \\
\hline 3 & $1 \cdot 7^{*}$ & $1 \cdot 0,2 \cdot 8$ & 1.4 & $0.8,2.5$ & 0.7 & $0.4,1 \cdot 3$ & 0.5 & $0 \cdot 2,1 \cdot 1$ \\
\hline 4 & 0.5 & $0.3,1.0$ & 1.0 & $0.5,1.9$ & 0.7 & $0.4,1.4$ & $1 \cdot 1$ & $0.5,2.4$ \\
\hline$P_{\text {trend }}$ & \multicolumn{2}{|c|}{0.16} & \multicolumn{2}{|c|}{0.57} & \multicolumn{2}{|c|}{0.48} & \multicolumn{2}{|c|}{0.64} \\
\hline \multicolumn{9}{|l|}{ Flavanones } \\
\hline 1 & 1.0 & Reference & & & 1.0 & Reference & & \\
\hline 2 & 1.3 & $0 \cdot 7,2 \cdot 3$ & & & 1.8 & $0.9,3.9$ & & \\
\hline 3 & 1.3 & $0 \cdot 7,2 \cdot 3$ & & & 1.3 & $0.6,3.0$ & & \\
\hline 4 & 1.3 & $0.7,2.4$ & & & 1.3 & $0.5,3.4$ & & \\
\hline$P_{\text {trend }}$ & \multicolumn{2}{|r|}{$0 \cdot 7,2 \cdot 4$} & \multicolumn{6}{|c|}{0.51} \\
\hline
\end{tabular}

${ }^{*} P<0.05$.

† Multivariate adjusted for energy, age at diagnosis, family history, non-steroidal anti-inflammatory drugs, aspirin, Mn, riboflavin, vitamin C, folate.

Our estimates of intake of flavonols and catechins are comparable with other tea-drinking populations and higher than non-tea-drinking countries such as Italy and America ${ }^{(20-25)}$, while flavanone intake was comparable with a Finnish study ${ }^{(23)}$, but lower than an Italian population study ${ }^{(28)}$. Intakes of flavonols, procyanidin and flavon-3-ols were higher among controls, but not cases, when compared with another recently published Scottish colorectal cancer study ${ }^{(29)}$. One reason for this may be a difference in the age distribution of subjects between the studies; in the present

Table 5. Association between non-tea flavonol intake by quartile of intake and colorectal cancers

(Odds ratios and $95 \%$ confidence intervals)

\begin{tabular}{|c|c|c|c|c|c|c|c|}
\hline & \multirow[b]{2}{*}{ Intake (mg) } & \multicolumn{2}{|c|}{ Colorectal cancer (261 cases) } & \multicolumn{2}{|c|}{ Colon cancer (186 cases) } & \multicolumn{2}{|c|}{ Rectal cancer (75 cases) } \\
\hline & & OR & $95 \% \mathrm{Cl}$ & OR & $95 \% \mathrm{Cl}$ & OR & $95 \% \mathrm{Cl}$ \\
\hline \multicolumn{8}{|c|}{ Quercetin } \\
\hline 1 & $<4.76$ & $1 \cdot 0$ & Reference & $1 \cdot 0$ & Reference & $1 \cdot 0$ & Reference \\
\hline 2 & $4 \cdot 77-6 \cdot 87$ & 0.8 & $0.5,1.3$ & 0.8 & $0.5,1.4$ & 0.7 & $0.4,1.5$ \\
\hline 3 & $6 \cdot 88-9.55$ & $0.6^{\star}$ & $0.4,1.0$ & 0.7 & $0.4,1 \cdot 2$ & 0.9 & $0.5,1.7$ \\
\hline 4 & $>9.56$ & $0 \cdot 6^{\star \star}$ & $0.4,0.9$ & $0.4^{*}$ & $0 \cdot 2,0 \cdot 8$ & 0.9 & $0.4,1.9$ \\
\hline $\begin{array}{r}P_{\text {trend }} \\
\text { Kaempf }\end{array}$ & & \multicolumn{2}{|c|}{$0 \cdot 01$} & \multicolumn{2}{|c|}{0.01} & \multicolumn{2}{|c|}{0.38} \\
\hline 1 & $<0.50$ & 1.0 & Reference & 1.0 & Reference & 1.0 & Reference \\
\hline 2 & $0.51-0.80$ & $1 \cdot 2$ & $0.7,1.9$ & $1 \cdot 2$ & $0.7,2 \cdot 1$ & $1 \cdot 1$ & $0 \cdot 5,2 \cdot 3$ \\
\hline 3 & $0 \cdot 81-1 \cdot 10$ & $1 \cdot 3$ & $0 \cdot 8,2 \cdot 0$ & $1 \cdot 2$ & $0 \cdot 7,2 \cdot 1$ & $1 \cdot 3$ & $0 \cdot 6,2 \cdot 8$ \\
\hline 4 & $>1 \cdot 11$ & $1 \cdot 1$ & $0 \cdot 6,2 \cdot 0$ & $1 \cdot 2$ & $0 \cdot 7,1 \cdot 1$ & 1.0 & $0 \cdot 4,2 \cdot 3$ \\
\hline $\begin{array}{r}P_{\text {trend }} \\
\text { Myriceti }\end{array}$ & & \multicolumn{2}{|c|}{0.94} & \multicolumn{2}{|c|}{0.98} & \multicolumn{2}{|c|}{0.98} \\
\hline 1 & $<0.04$ & $1 \cdot 0$ & Reference & $1 \cdot 0$ & Reference & 1.0 & Reference \\
\hline 2 & $0.05-0.20$ & 0.6 & $0.4,0.9$ & $0.6^{*}$ & $0.4,1.0$ & 0.6 & $0 \cdot 3,1 \cdot 2$ \\
\hline 3 & $0.21-0.44$ & 0.7 & $0.5,1 \cdot 1$ & $0.6^{*}$ & $0.3,0.9$ & $1 \cdot 2$ & $0 \cdot 6,2 \cdot 3$ \\
\hline 4 & $>0.45$ & 0.7 & $0.5,1.1$ & 0.7 & $0.4,1 \cdot 2$ & 0.8 & $0.4,1 \cdot 7$ \\
\hline$P_{\text {trend }}$ & & \multicolumn{2}{|c|}{0.27} & \multicolumn{2}{|c|}{0.13} & \multicolumn{2}{|c|}{0.94} \\
\hline
\end{tabular}

* $P<0.05,{ }^{* *} P<0.01$.

†Adjusted for energy, age, education, family history, non-steroidal anti-inflammatory drugs, aspirin, vitamin $\mathrm{C}$, folate, fruit and vegetables. 
study both cases and controls were on average 6 years older than in the other study. Though a relatively small difference, in the UK tea consumption by the 64- to 74-year age group is $25 \%$ higher than by the 50 - to 64 -year-olds ${ }^{(39)}$.

The results of our regional North East of Scotland study are only partially consistent with those of the larger Scottish study of Theodoratou et al. ${ }^{(29)}$. In their study, increasing total dietary flavonol (OR $\left.0.73 ; P_{\text {trend }}<0.02\right)$, quercetin (OR $0.68 ; P_{\text {trend }}<0.001$ ), catechin (OR 0.68; $P_{\text {trend }}<0.001$ ), epicatechin (OR $\left.0.74 ; P_{\text {trend }}<0.05\right)$ and procyanidins (OR $\left.0.78 ; P_{\text {trend }}<0.05\right)$ intake significantly reduced the risk of developing colorectal cancer, while no effect was found for total flavanones or flavon-3-ols. The latter result is consistent with our findings; however, in contrast to Theodoratou et al. ${ }^{(29)}$ we did not observe a trend in risk with total flavonols, catechin or epicatechin intakes and colorectal cancer. One explanation may be that our findings may reflect the smaller sample size or the different age distribution of subjects. Alternatively it may be that, due to regional variations in diet within Scotland, our population drank more tea and consumed less fruit and vegetables, providing relatively lower non-tea sources of flavonoids. Our findings with regard to non-tea flavonoid intake reflect those of a large Italian case-control study ${ }^{(28)}$. Researchers in the Italian study identified a positive trend against colorectal cancers with increasing intake of flavonols (OR 0.64; $P_{\text {trend }}<0.001$ ), but not flavon-3-ols or flavanones in a population with high fruit and vegetable intake and infrequent tea consumption. Each of the now three case-control studies have utilised recently compiled and relatively comprehensive flavonoid composition databases, one developed in the UK reflecting the flavonoid content of foods commonly consumed in Europe ${ }^{(26)}$ and the $\mathrm{USA}^{(40)}$ unlike the previously published cohort studies, in which an earlier more limited Dutch database with regional additions were employed ${ }^{(27,41-43)}$. Of earlier studies, only one reported significant associations between flavonoid intake and risk of colorectal cancer ${ }^{(23)}$. The Iowa Women's Health study, a cohort of 34651 postmenopausal women, observed a significant inverse association between total flavon-3-ol intake and rectal cancer. The present study did not replicate these findings even when the analysis was limited to females only, but there were only twenty-eight women with rectal cancer in the present study. Black tea is not commonly consumed in America, and comparison of the US intake with the Scottish non-tea flavon-3-ol intake indicates that the Scottish non-tea intake $(>8.5 \mathrm{mg} / \mathrm{d})$ may be too low to detect a protective effect.

The lack of an association between black tea consumption and colorectal cancer risk is in agreement with previous findings ${ }^{(3)}$. As total dietary flavonol, procyanidin and flavon-3-ol intakes were highly correlated with black tea consumption, a case has been made to study total and non-tea flavonoid intake separately ${ }^{(24)}$. Assessment of these independent associations with risk of developing colorectal cancer suggests that flavonoids from dietary sources other than tea may modulate colorectal cancer risk.

Previous dietary flavonoid intake assessment in the North East of Scotland revealed that the main dietary sources of flavonoids were tea (46\% of the intake), onions $(14 \%)$, apples $(10 \%)$ and processed foods and beverages $(13 \%)$ for flavonols ${ }^{(44)}$. This suggests that flavonol intake from a diverse combination of fruit- and vegetable-based foods may be responsible for the observed inverse association.

Individual flavonoid compounds and their dietary sources have differing relative bioavailability ${ }^{(45)}$. Consequently, when assessing several flavonoid subclasses in relation to disease, the sources and types of flavonoids could have different potential relationships with colorectal cancer. The structureactivity relationship of flavonoids provides an analytical example to support this observation. Ranking of the ability of flavonoids to scavenge different reactive oxygen and nitrogen species in both aqueous and lipophilic environments indicates that quercetin is a potent antioxidant in vitro ${ }^{(46,47)}$. As large amounts of flavonoids remain unabsorbed in the lower gastrointestinal tract, they may exert their antioxidant effects at that site ${ }^{(19)}$. An imbalance between cellular levels of reactive oxygen species and antioxidants can result in damage to DNA, leading to mutation and dysregulation of oncogenes or tumour-suppressor genes ${ }^{(46)}$. Quercetin, as one of the most potent antioxidants in vitro, may act to minimise mutations. Suppression of cellular proliferation may also be structure dependent. For example, quercetin, but not catechin, can suppress proliferation of colon cancer cell lines ${ }^{(48)}$. More recently quercetin has been reported to modulate cell growth signalling pathways ${ }^{(16,49-52)}$. Finally, chronic inflammation is an important target for preventive measures against colon cancer, with non-steroidal anti-inflammatory drugs and aspirin, for example, being thought to reduce risk by up to $50 \%{ }^{(53,54)}$. This is principally achieved through modulation of arachidonic acid release and its subsequent metabolism by cyclo-oxygenase and lipoxygenase, mediators of the inflammatory response ${ }^{(54)}$. Flavonoids are known to modulate expression of these enzymes with quercetin, in particular, inhibiting their activity ${ }^{(8,12,13)}$.

The inverse association observed between colorectal cancer risk and non-tea flavonol intake but not tea flavonoids in the present study is interesting and highlights the importance of assessing their different dietary sources in relation to disease risk. It is only the non-tea dietary sources of flavonols that are significantly associated with a protective effect against colorectal cancer. This may circumstantially suggest that other dietary factors in fruit and vegetables in addition to flavonols have important roles in preventing the pathogenesis of the disease. Further investigation of the effect of flavonols from different fruit and vegetables and their processed products is required to determine whether the observed association is due to flavonols per se or to the other as yet unidentified components of fruit and vegetables which are co-associated with flavonoids. Intervention studies comparing the effects of individual flavonoids with flavonoid-rich diets may be required to elucidate whether the main protective effects are actually due to these phytochemicals.

\section{Acknowledgements}

The original case-control study was funded by a grant from the National Hospitals Trust. The flavonoid analysis was financially supported by the Scottish Executive Environment and Rural Affairs Department and the Food Standards Agency.

J. A. M. K. led the development of the flavonoid database and analysis, contributed to the interpretation of results and 
drafted the manuscript. L. S. and J. L. were the principal investigators of the case-control study, contributed to the statistical analysis and interpretation of the results, and contributed to the paper. G. G. D. and G. McN. supervised the flavonoid analysis, and contributed to the interpretation of the results and the paper. We thank Graeme McHardy, Diane Thom, Seonaidh Cotton, Nigel Brockton and David Grubb for assistance with development of study procedures, data collection and analysis.

None of the authors has any conflict of interest.

\section{References}

1. World Health Organization (2003) Diet, Nutrition and the Prevention of Chronic Diseases. Joint WHO/FAO Expert Consultation. WHO Technical Report Series no. 916. Geneva: WHO.

2. Information Services Division NHS National Services Scotland (2009) Cancer in Scotland Summary. http://www.isdscotland. org/isd/1425.html\#Colorectal\%20cancer

3. World Cancer Research Fund \& American Institute for Cancer Research (2007) Food, Nutrition, Physical Activity, and the Prevention of Cancer: a Global Perspective. Washington, DC: AICR.

4. Duijnhoven FJB, De Mesquita HB, Ferrari P, et al. (2009) Fruit, vegetables, and colorectal cancer risk: the European Prospective Investigation into Cancer and Nutrition. Am J Clin Nutr 89, $1441-1452$.

5. Smith-Warner SA, Genkinger J \& Giovannucci E (2000) Fruit and vegetable intake and cancer. In Nutritional Oncology, pp. 153-184 [D Herber, GL Blackburn and VLM Go, editors]. San Diego: Academic Press Inc.

6. Nichenametla SN, Taruscio TG, Barney DL, et al. (2006) A review of the effects and mechanisms of polyphenolics in cancer. Crit Rev Food Sci Nutr 46, 161-183.

7. Lambert JD, Hong J, Yang G, et al. (2005) Inhibition of carcinogenesis by polyphenols: evidence from laboratory investigations. Am J Clin Nutr 81, Suppl., 284S-291S.

8. Surh YJ, Kundu JK, Na HK, et al. (2005) Redox sensitive transcription factors as prime targets for chemoprevention with anti-inflammatory and antioxidative phytochemicals. J Nutr 135, 2993S-3001S.

9. Duthie SJ, Johnson W \& Dobson VL (1997) The effect of dietary flavonoids on DNA damage (strand breaks and oxidized pyrimidines) and growth in human cells. Mutat Res 390, $141-151$.

10. Sánchez de Medina F, Vera B, Gálvez J, et al. (2002) Effect of quercitrin on the early stages of hapten induced colonic inflammation in the rat. Life Sci 70, 3097-3108.

11. Kim HP, Mani I, Iversen L, et al. (1998) Effects of naturally occurring flavonoids and biflavonoids on epidermal cyclooxygenase and lipoxygenase from guinea-pigs. Prostaglandins Leukot Essent Fatty Acids 58, 17-24.

12. Kim HP, Son KH, Chang HW, et al. (2004) Anti-inflammatory plant flavonoids and cellular action mechanisms. J Pharmacol Sci 96, 229-245.

13. Peng G, Dixon DA, Muga SJ, et al. (2006) Green tea polyphenol (-)-epigallocatechin-3-gallate inhibits cyclooxygenase-2 expression in colon carcinogenesis. Mol Carcinog 45, 309-319.

14. Daskiewicz JB, Depeint F, Viornery L, et al. (2005) Effects of flavonoids on cell proliferation and caspase activation in a colonic cell line HT 29, SAR study. J Med Chem 48, $2790-2804$.
15. Franke AA, Custer LJ, Cooney RV, et al. (2002) Inhibition of colonic aberrant crypt formation by the dietary flavonoids (+)-catechin and hesperidin. Adv Exp Med Biol 505, 123-133.

16. Gosse F, Guyot S, Roussi S, et al. (2005) Chemopreventive properties of apple procyanidins on human colon cancer derived metastatic SW620 cells and in a rat model of colon carcinogenesis. Carcingogenesis 26, 1291-1295.

17. Williams RJ, Spencer JPE \& Rice-Evans C (2004) Flavonoids: antioxidants or signalling molecules? Free Rad Biol Med 36, $838-849$.

18. Van Dross R, Xue Y, Knudson A, et al. (2003) The chemopreventive bioflavonoid apigenin modulates signal transduction pathways in keratinocyte and colon carcinoma cell lines. J Nutr 133, 3800S-3804S.

19. Halliwell B, Zhao KC \& Whiteman M (2000) The gastrointestinal tract: a major site of antioxidant action? Free Radic Res 33, 819-830.

20. Lin J, Zhang SM, Wu K, et al. (2006) Flavonoid intake and colorectal cancer risk in men and women. Am J Epidemiol 164, 644-651.

21. Hirvonen T, Virtamo J, Korhonen P, et al. (2001) Flavonol and flavone intake and the risk of cancer in male smokers (Finland). Cancer Causes Control 12, 789-796.

22. Knekt P, Jarvinen R, Seppanen R, et al. (1997) Dietary flavonoids and the risk of lung cancer and other malignant neoplasms. Am J Epidemiol 146, 223-230.

23. Knekt P, Kumpulainen J, Järvinen R, et al. (2002) Flavonoid intake and risk of chronic disease. Am J Clin Nutr 76, 560-568.

24. Arts ICW, Hollman PCH, de Mesquita HBB, et al. (2001) Dietary catechins and epithelial cancer incidence: the Zutphen elderly study. Int J Cancer 92, 298-302.

25. Arts ICW, Jacobs DR Jr, Gross M, et al. (2002) Dietary catechins and cancer incidence among postmenopausal women: the Iowa Women's Health Study (United States). Cancer Causes Control 13, 373-382.

26. Kyle JAM \& Duthie GG (2006) Flavonoids in foods. In Flavonoids; Chemistry, Biochemistry and Health Implications, pp. 219-263 [OM Andersen and K Markham, editors]. New York: Taylor and Francis Group.

27. Hertog MGL, Hollman PCH \& Katan MB (1992) Content of potentially anticarcinogenic flavonoids of 28 vegetables and 9 fruits commonly consumed in The Netherlands. J Agric Food Chem 40, 2379-2783.

28. Rossi M, Negri E, Talamini R, et al. (2006) Flavonoids and colorectal cancer in Italy. Cancer Epidemiol Biomarkers Prev 15, 1555-1558

29. Theodoratou E, Kyle J, Cetnarskyj R, et al. (2007) Dietary flavonoids and the risk of colorectal cancer. Cancer Epidemiol Biomarkers Prev 16, 684-693.

30. Scottish Health Service Advisory Council (1993) The Scottish Diet: Report of a Working Party to the Chief Medical Officer for Scotland. Edinburgh: Scottish Office Home and Health Department.

31. McKie L, MacInnes A, Hendry J, et al. (2000) The food consumption patterns and perceptions of dietary advice of older people. J Hum Nutr Diet 13, 173-183.

32. Turrini A, Saba A, Perrone D, et al. (2001) Food consumption patterns in Italy: the INN-CA study 1994-1996. Eur J Clin Nutr 55, 571-588.

33. Little J, Sharp L, Masson LF, et al. (2006) Colorectal cancer and genetic polymorphisms of CYP1A1, GSTM1 and GSTT1: a case-control study in the Grampian region of Scotland. Int J Cancer 119, 2155-2164.

34. Food Standards Agency (2002) McCance and Widdowson's The Composition of Foods, 6th summary ed. Cambridge, UK: Royal Society of Chemistry. 
35. Kyle J, Masson L, Duthie GG, et al. (2002) Estimating dietary flavonoid intake: comparison of a semi-quantitative food frequency questionnaire with 4-day weighed diet records in a Scottish population. Proc Nutr Soc 61, 69A.

36. Masson LF, McNeill G, Tomany JO, et al. (2003) Statistical approaches for assessing the relative validity of a food frequency questionnaire: use of correlation coefficients and the $\kappa$ statistic. Public Health Nutr 6, 313-321.

37. Willett W (1998) Implications of total energy intake for epidemiologic analyses. In Nutritional Epidemiology, 2nd ed., pp. 288-291 [W Willett, editor]. New York: Oxford University Press.

38. Macfarlane TV, Gray RJM, Kincey J, et al. (2007) Factors associated with the temporomandibular disorder, pain dysfunction syndrome (PDS): Manchester case-control study. Oral Dis 7, 321-330.

39. Department for Environment, Food and Rural Affairs (2007) Family Food - Report on the Expenditure of Food Survey. National Statistics Publication. London: The Stationery Office.

40. United States Department of Agriculture (2003) USDA Database for the Flavonoid Content of Selected Foods. Beltsville, MD: USDA.

41. Hertog MGL, Hollman PCH \& van de Putte B (1993) Content of potentially anticarcinogenic flavonoids of tea infusions, wines and fruit juices. J Agric Food Chem 41, 1242-1246.

42. Arts IC, van de Putte B \& Hollman PC (2000) Catechin contents of foods commonly consumed in The Netherlands. 1. Fruits, vegetables, staple foods, and processed foods. J Agric Food Chem 8, 1746-1751.

43. Arts IC, van De Putte B \& Hollman PC (2000) Catechin contents of foods commonly consumed in The Netherlands. 2. Tea, wine, fruit juices, and chocolate milk. J Agric Food Chem 48, 1752-1757.
44. Kyle JAM (2003) Flavonoids in health and disease. PhD Thesis, University of Aberdeen, UK

45. Hollman PCH \& Arts ICW (2000) Flavonols, flavones and flavanols - nature, occurrence and dietary burden. J Sci Food Agric 80, 1081-1093.

46. Duthie GG, Duthie SJ \& Kyle JAM (2000) Plant polyphenols in cancer and heart disease: implications as nutritional antioxidants. Nutr Res Rev 13, 79-106.

47. Heim KE, Tagliaferro AR \& Bobilya DJ (2002) Flavonoid antioxidants: chemistry, metabolism and structure-activity relationships. J Nutr Biochem 13, 572-584.

48. Agullo G, Gamet L, Besson C, et al. (1994) Quercetin exerts a preferential cytotoxic effect on active dividing colon carcinoma HT29 \& Caco-2 cells. Cancer Lett 87, 55-63.

49. Kim WK, Bang MH, Kim ES, et al. (2005) Quercetin decreases the expression of ErbB2 and ErbB3 proteins in HT-29 human colon cancer cells. J Nutr Biochem 16, 155-162.

50. Park CH, Chang JY, Hahm ER, et al. (2005) Quercetin, a potent inhibitor against $\beta$-catenin/Tcf signalling in SW480 colon cancer cells. Biochem Biophys Res Commun 328, 227-234.

51. Mouat MF, Kolli K, Orlando R, et al. (2005) The effects of quercetin on SW480 human colon carcinoma cells: a proteomic study. Nutr J 4, 11.

52. Wenzel U, Hertzog A, Kuntz S, et al. (2004) Protein expression profiling identifies molecular targets of quercetin as a major dietary flavonoid in human colon cancer cells. Proteomics 4, $2160-2174$

53. Dubé C, Rostom A, Lewin G, et al. (2007) The use of aspirin for primary prevention of colorectal cancer: a systematic review prepared for the U.S. Preventive Services Task Force. Ann Intern Med 146, 365-375.

54. Vane JR \& Botting RM (1998) Anti-inflammatory drugs and their mechanism of action. Inflamm Res 47, S78-S87. 